\title{
Pain and the Emotional Brain: Affective Rather than Cognitive Processes Drive the Cortical Encoding of Pain
}

\author{
Astrid Mayr ${ }^{1}$, Anne Stankewitz ${ }^{1}$, Stephanie Irving ${ }^{1}$, Viktor Witkovsky ${ }^{3}$, Enrico Schulz ${ }^{1,2,4}$ \\ ${ }^{1}$ Department of Neurology, Ludwig-Maximilians-Universität München, Munich, Germany \\ ${ }^{2}$ Wellcome Centre for Integrative Neuroimaging, FMRIB, Nuffield Department of Clinical \\ Neurosciences, University of Oxford, Oxford, UK \\ ${ }^{3}$ Department of Theoretical Methods, Institute of Measurement Science, Slovak Academy \\ of Sciences, Bratislava, Slovak Republic \\ ${ }^{4}$ Department of Medical Psychology, Ludwig-Maximilians-Universität München, Munich, \\ Germany
}

Corresponding author:

Enrico Schulz

Ludwig-Maximilians-Universität München

Neurologische Klinik und Poliklinik

A: Fraunhoferstr. 20, 82152 Martinsried, Germany

O: +4989440074826

E: es@pain.sc 


\section{ABSTRACT}

The experience of pain has been dissociated into two interwoven aspects: a sensory-discriminative aspect assessed in ratings of pain intensity and an affective-motivational aspect assessed in ratings of unpleasantness. In a pain attenuation paradigm, participants were asked to evaluate applied cold pain. The majority of the trials showed a distinct rating: Some trials were rated higher for unpleasantness, others were rated higher for intensity. Using linear mixed effect models on single trials, we related the variable difference between unpleasantness and intensity ratings to functional MRI data. The direct comparison revealed a stronger relationship between cortical data and pain ratings for unpleasantness. No region showed a stronger effect for pain intensity. The present study underlines the importance of the emotional-affective aspects of pain-related cortical processes in the brain. These findings reflect the biological function of the pain system to prevent harm and to preserve the physical integrity of the body. 


\section{INTRODUCTION}

The experience of pain has been dissociated into sensory-discriminative and affective-motivational aspects ${ }^{1}$, which are assessed as subjective ratings of pain intensity and pain unpleasantness ${ }^{2-4}$. Both descriptors of pain share a conceptual core variance and аге therefore often highly correlated: very intense pain is associated with higher levels of unpleasantness ${ }^{5,6}$. The experience of pain unpleasantness is considered to exhibit a particularly strong influence on well-being ${ }^{7,8}$, as it has been found to be tightly connected to pain catastrophising ${ }^{7}$.

Studies exploring the cortical underpinnings of pain unpleasantness are scarce and non-specific as they implicitly include the shared variance with pain intensity ${ }^{9,10}$. The conceptual and empirical proximity of both aspects has made a differential investigation of pain intensity and pain unpleasantness challenging. A series of early PET studies attempted to independently modulate pain intensity and pain unpleasantness using hypnosis. For pain unpleasantness, the group reported a specific modulation in the anterior cingulate cortex (ACC) ${ }^{6,11}$. This region was also active to a lesser extent in the low-unpleasant condition, but was not found in any of the conditions of the intensity modulation. This absence of activity in this ACC region in the pain intensity study, as well as the lack of a direct comparison with pain unpleasantness, leaves some questions regarding distinct cortical processing of pain intensity and pain affect ${ }^{12}$.

Nonetheless, despite a high correlation between both subjective pain aspects, it is indeed possible to dissociate the cortical underpinnings of pain intensity and pain unpleasantness in a within-subject design without the need for any modulatory intervention. To this end, we aimed to investigate the brain regions that differentially process pain intensity and pain unpleasantness. As a major advantage, both aspects are explored in relation to the very same underlying cortical data; each trial has been evaluated for either descriptor of the subjective experience of pain. The majority of the trials indeed differed and enabled us to elucidate which aspect of pain perception is more deeply rooted in the human brain.

\section{RESULTS}

\section{Behavioural data}

The single-trial pain ratings varied substantially across trials; we recorded an average of 34 $( \pm 14)$ for pain intensity and of $31( \pm 17)$ for pain unpleasantness. Both aspects of pain perception were highly correlated $(r=0.86, p<0.01$, Figure 1$)$. Nonetheless, the majority of the trials exhibited a distinct rating for intensity and unpleasantness (75\%, Figure 1 ). 


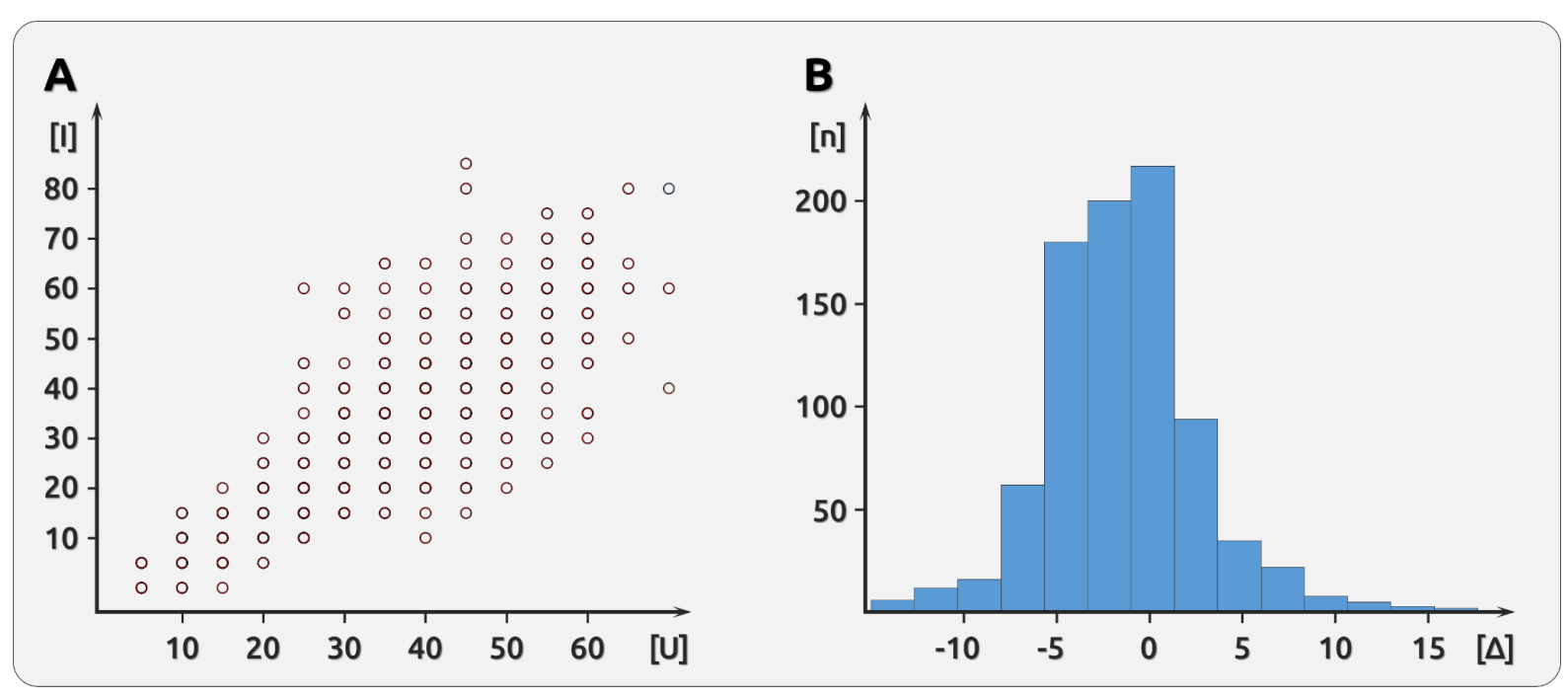

Figure 1. The left side (A) shows the correlation between pain intensity [I] and pain unpleasantness $[\mathrm{U}]$. The correlation coefficient is $r=0.86$. Nevertheless, the ratings for both aspects were identical only for a minority of the trials. The histogram (B) shows the difference between pain intensity ratings and pain unpleasantness ratings; most trials $(\mathbf{7 5} \%)$ exhibited different ratings. For some trials, pain intensity was rated higher (negative $\Delta$ ), for others pain unpleasantness was rated higher (positive $\Delta$ ).

\section{Imaging data}

The results indicate a stronger positive relationship for pain unpleasantness in regions that are known to be involved in pain processing: the bilateral anterior and posterior insula, postcentral regions, regions in the frontal cortex, the paracingulate regions, and the nucleus ruber. Additionally, we found results for the negative gradient, indicating a more positive relationship for pain intensity for the retrosplenial cortex, the precuneous, the posterior cingulate cortex, the occipital cortex, the amygdala, and the middle frontal gyrus. However, these negative results occur exclusively in brain regions that are suppressed in response to pain. Therefore, they reflect a stronger negative relationship between pain ratings and brain activity for unpleasantness (Figure 2).

Please note that the differences are in a gradual fashion; due to the fact that both aspects of pain evaluation are highly correlated, they are considered to be commonly processed in the same cortical regions, but to a different extent. All trials on intensity and unpleasantness are relying on the same cortical responses.

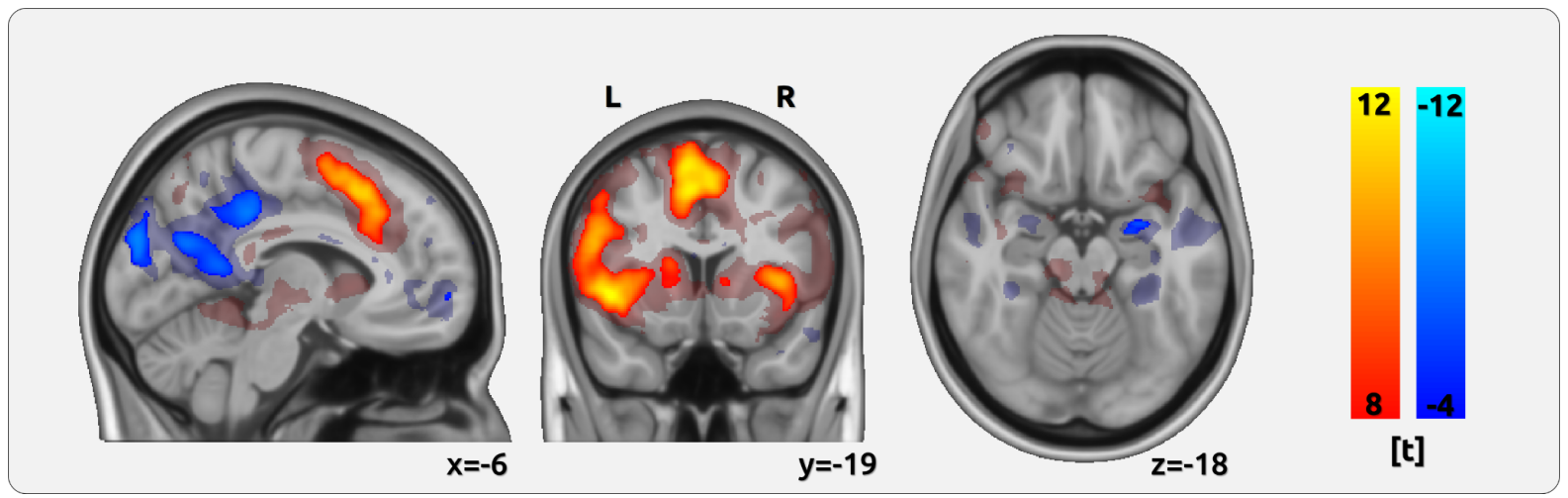

Figure 2. Several cortical regions reflect the gradual differences between pain intensity and pain unpleasantness. Warm colours indicate regions that show a more positive relationship for pain unpleasantness; cold colours indicate brain regions that show a stronger negative 
relationship for pain unpleasantness. In order to better separate the different regions we increased the statistical threshold beyond the significance level $(t>4.9)$ for the positive effects.

\begin{tabular}{|c|c|c|c|c|}
\hline max t-value & $x$ & $y$ & z & region \\
\hline-8.45 & 1 & -69 & 25 & $\begin{array}{l}\text { Retrosplenial Cortex, Precuneous, } \\
\text { Posterior Cingulate Cortex }\end{array}$ \\
\hline 12.4 & 42 & 13 & 12 & $\begin{array}{l}\text { Insular Cortex, Inferior Frontal } \\
\text { Cortex, Striatum }\end{array}$ \\
\hline 12.5 & 4 & 16 & 48 & $\begin{array}{l}\text { Superior Frontal Gyrus, } \\
\text { Juxtapositional Gyrus, } \\
\text { Paracingulate Gyrus }\end{array}$ \\
\hline 12.3 & -32 & 13 & 3 & Insular Cortex, Striatum \\
\hline 10.8 & -33 & -50 & 46 & Superior Parietal Lobe \\
\hline 10.6 & 40 & -44 & 46 & Superior Parietal Lobe \\
\hline-8.34 & 26 & 31 & 43 & Middle Frontal Gyrus \\
\hline 10.2 & -24 & -5 & 56 & Superior Frontal Gyrus \\
\hline 9.73 & 38 & 47 & 20 & Frontal Pole \\
\hline 7.08 & -1 & -22 & 28 & Posterior Cingulate Cortex \\
\hline 9.1 & 29 & -1 & 55 & Middle Frontal Gyrus \\
\hline 9.37 & -37 & -23 & 56 & Postcentral Gyrus \\
\hline-5.35 & 4 & 59 & -5 & Frontal Pole \\
\hline 9.65 & -47 & 3 & 34 & Precentral Gyrus \\
\hline-5.87 & 58 & -55 & 4 & Middle Temporal Gyrus \\
\hline 10.2 & 8 & -54 & -10 & Cerebellum \\
\hline-6.74 & 26 & -5 & -18 & Amygdala \\
\hline 8.79 & -42 & 28 & 30 & Middle Frontal Gyrus \\
\hline 6.86 & -9 & -22 & -7 & Brainstem, ncl. ruber \\
\hline
\end{tabular}

Table 1. Differences in the encoding of pain intensity and pain unpleasantness. Positive effects indicate a stronger positive relationship for unpleasantness; negative relationships indicate a stronger negative relationship for unpleasantness (all p<0.01, PALM corrected).

\section{DISCUSSION}

In the present study, we were investigating the cortical underpinnings of pain intensity and pain unpleasantness. Despite the fact that each shares the same fundamental core, we took advantage of the sufficient variability, which allowed us to investigate cortical functions related to the subjective experience of pain in a within-subject design at single-trial level. Overall, we revealed a stronger relationship for the pain-related cortical 
processes regarding the affective aspect compared to the sensory-discriminative aspect, suggesting that the emotional-affective aspect of pain is more deeply rooted in the human brain.

Regions that increased activity in response to pain had a more positive effect for unpleasantness, and regions that exhibited decreased activity showed a more negative effect for unpleasantness. Consequently, unpleasantness ratings may reflect the more direct and intuitive processing of emotions in the human brain rather than the more complex and "minded" evaluation of pain intensity. Due to the methodological differences of previous work and their inherent weaknesses, the present study is not comparable to previous imaging studies ${ }^{11,13}$. However, our results underline other research indicating the preferential processing of pain-related emotions in the human brain ${ }^{14,15}$. It has been suggested that the feeling of unpleasantness belongs to the initial part of the noxious sensation and occurs before any conscious and cognitive processing of the sensation ${ }^{16}$. Therefore, the stronger effect of pain unpleasantness may better reflect the biological function of the pain system to prevent harm and to preserve the physical integrity of the body ${ }^{17}$.

Indeed, there is a large framework of research that supports the notion that emotional processes are tightly associated with pain processing in the brain. Higher pain affect has been found to increase autonomic responses, such as skin conductance ${ }^{18,19}$, which in turn were found to encode pain-related cortical responses ${ }^{20,21}$. Potentially emotion-related autonomic responses were found to be more tightly related to cortical activity than ratings of pain intensity ${ }^{20}$.

Our study complements previous findings by Zeidan and colleagues who shed light on the individual abilities to modulate pain intensity and pain unpleasantness through meditation 13. The better performing participants were able to make use of specific subunits of the pain modulation system. The participants that exhibited a better performance in the attenuation of pain unpleasantness, modulated the activity of the orbitofrontal cortex ${ }^{13}$. This region that has been suggested to serve as a major hub of emotional modulation ${ }^{22}$. In a similar vein, the hub that modulates the ability to attenuate pain intensity through meditation could be located in the anterior insula ${ }^{13}$. Depending on the pain attenuation strategy, we would assume the existence of further cortical hubs that are differently utilised by individual subjects and which alter pain intensity and pain unpleasantness ${ }^{23,24}$.

Taken together, the present study shows that pain unpleasantness is more tightly related to cortical processing than the cognitive evaluation of pain intensity. These findings generalise to all brain regions that exhibit pain-related responses, irrespective of whether they increase or decrease their activity. Consequently, studies utilising the evaluation of painful stimulation are suggested to assess pain unpleasantness rather than pain intensity. These suggestions may also be expanded to investigations of endogenous pain in chronic patients. Future studies need to shed further light on either aspect of pain evaluation under specific psychological conditions, and we hypothesise temporal differences in the cascade of pain-related cortical processes: the cortical processes of pain unpleasantness evaluation are processed faster and may occur before the cognitive and "minded" cortical processes of pain intensity evaluation. 


\section{ACKNOWLEDGEMENTS}

This project was funded by the "Deutsche Forschungsgemeinschaft" (DFG, SCHU 2879/1-2)".

\section{DECLARATION OF INTERESTS}

All authors declare no conflict of interest.

\section{DATA AVAILABILITY}

The data will be made available via the Open Science Framework (https://osf.io/tbc2u/).

\section{CODE AVAILABILITY}

No custom code has been developed for this study.

\section{MATERIALS AND METHODS}

Subjects. 20 healthy subjects (16 female/4 male, mean age $27 \pm 5$ years) were included in the study. All subjects gave written informed consent. The study was approved by the Medical Sciences Interdivisional Research Ethics Committee of the University of Oxford and conducted in conformity with the Declaration of Helsinki.

The experimental procedure has been described in detail in our previous publications ${ }^{23,24}$. The experiment consisted of four conditions across four separate blocks, where each block comprised 12 trials from the same condition. In all conditions and trials, the subjects received cold pain stimuli on the dorsum of their left hand delivered by a thermode (Pathway II; Medoc Ltd, Israel). The first condition of the experiment was always the unmodulated pain condition. The subsequent three conditions were counterbalanced blocks of pain attenuation: (A) an attentional shift, (B) an imaginal strategy, and (C) a non-imaginal reinterpretation. After each trial, the subjects were prompted to rate pain intensity and pain unpleasantness within $10 \mathrm{~s}$. The scale ranged between 0 and 100 in steps of 5 points. The endpoints of the scale were determined as no pain (0) and the maximum pain the subjects were willing to tolerate (100). There were a total of 48 trials during fMRI recording. The $40 \mathrm{~s}$ of painful stimulation were preceded by a rest period of 10 $\mathrm{s}$ at $38^{\circ} \mathrm{C}$ thermode temperature.

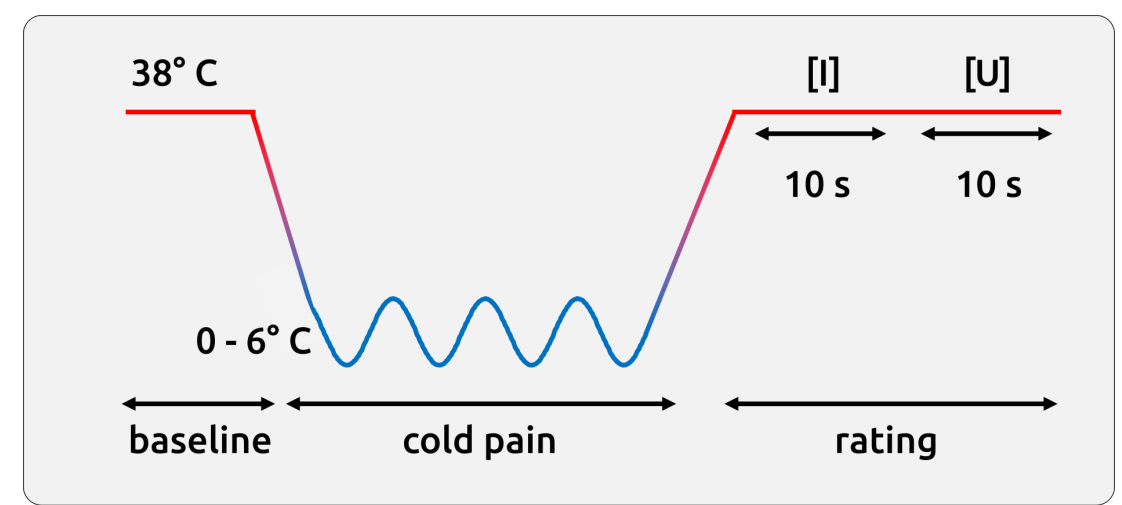

Supplementary Figure 1: Schematic time course of a single trial. After 10 $s$ of rest, painful stimulation was applied for 40 s. The pain was rated regarding intensity ([I], 10 s) and unpleasantness ([U], 10 s). 
Data Acquisition. Imaging data were acquired on a 7 tesla Siemens MRI scanner using parallel image acquisition (GRAPPA, factor $=2$ ). In order to cover the whole brain, each of the 1768 functional echo-planar imaging (EPI) volumes comprised 34 axial slices of $2 \mathrm{~mm}$ thickness and $2 \times 2 \mathrm{~mm}$ in-plane resolution with a $1 \mathrm{~mm}$ gap between slices. The repetition time (TR) was $1.96 \mathrm{~s}$, the echo time (TE) was $25 \mathrm{~ms}$ (flip angle $90^{\circ}$ ), the field of view (FOV) was $220 \times 220 \mathrm{~mm}$, and the matrix size was $110 \times 110$ pixels. A T1-weighted structural image (isotropic $1 \mathrm{~mm}^{3}$ voxel) was acquired for the registration of the EPI to the MNI (Montreal Neurological Institute) template.

Imaging analysis. The data were pre-processed and analysed with FSL ${ }^{25}$ and Matlab (version R2020a, The Mathworks, USA). The preprocessing consisted of brain extraction, high-pass filtering with a frequency cutoff of $1 / 90 \mathrm{~Hz}$, a correction for head motion during scanning, spatial normalisation to the MNI template, and spatial smoothing (6 mm FWHM). The data were further semi-automatically cleaned of artefacts with MELODIC ${ }^{26}$. Beta coefficients that represent the magnitude of cortical activity for each trial were computed in FEAT ${ }^{24}$.

Statistical analysis. Using Linear Mixed Effects models 27,28, we aimed to differentiate the relationship between the two aspects of pain ratings and cortical activity. Across all trials we created a vector representing the single trial difference between pain intensity and pain unpleasantness (UI_gradient). Differences between pain intensity and pain unpleasantness occurred in $75 \%$ of trials. For the intensity trials we created a new vector by subtracting the ratings from the mean between intensity and unpleasantness for the trial. We used the analogue procedure for the unpleasantness trials:

\section{(1) UI_gradient = [Unpleas-Intens/2+Unpleas $/ 2 ;$ Intens-Intens $/ 2+$ Unpleas $/ 2]$}

The statistical model is expressed in Wilkinson notation ${ }^{29}$; the included fixed effect of interest (UI_gradient:fmri) describes the magnitudes of the population common intercept and the population common slopes for the relationship between cortical data and the single trial differences in pain perception. The added random effect (i.e. 1/subject) models the specific intercept differences for each subject (e.g. subject-specific differences in fMRI beta coefficients):

\section{(2) pain fmri + UI_gradient:fmri + (1/subject)}

Analysis was restricted to pain-related brain regions; we created a 3D mask consisting of the group result of the analysis in FEAT contrasting the pure pain trials with the baseline period ( $t>2)$. To correct for multiple comparisons we applied a randomisation approach. Behavioural data were shuffled and the entire analysis was repeated 5000 times within the boundaries of the 3D brain mask. The highest absolute t-values of each repetition across the whole volume were extracted. This procedure resulted in right-skewed distribution for each condition. Based on these distributions, the statistical thresholds were determined using the "palm_datapval" function publicly available in PALM ${ }^{30}$. 


\section{REFERENCES}

1 Melzack R, Casey KL. Sensory, motivational, and central control determinants of pain: a new conceptual model in pain. In: Kenshalo DR (ed). . Springfield, Ill., 1968, pp 423-443.

2 Kunz $M$, Lautenbacher $S$, LeBlanc $N$, Rainville $P$. Are both the sensory and the affective dimensions of pain encoded in the face? Pain 2012; 153: 350-358.

3 Rainville P, Carrier B, Hofbauer RK, Bushnell CM, Duncan GH. Dissociation of sensory and affective dimensions of pain using hypnotic modulation. Pain 1999; 82: 159-171.

4 Perlman DM, Salomons TV, Davidson RJ, Lutz A. Differential effects on pain intensity and unpleasantness of two meditation practices. Emotion 2010; 10: 65-71.

5 Coghill RC, Sang CN, Maisog JM, ladarola MJ. Pain intensity processing within the human brain: a bilateral, distributed mechanism. J Neurophysiol 1999; 82: 1934-1943.

6 Hofbauer RK, Rainville P, Duncan GH, Bushnell MC. Cortical representation of the sensory dimension of pain. J Neurophysiol 2001; 86: 402-411.

7 Gagnon-Dolbec A, Fortier M, Cormier S. Pain intensity and pain unpleasantness in triathletes: A study examining their associations with pain catastrophizing and pain expectations. Psychol Sport Exerc 2021; : 101928.

8 Shriver A. The unpleasantness of pain for humans and other animals. Philosophy of Pain. 2018; : 147-162.

9 Zunhammer $M$, Geis S, Busch V, Eichhammer $\mathrm{P}$, Greenlee MW. Pain modulation by intranasal oxytocin and emotional picture viewing - a randomized double-blind fMRI study. Scientific Reports. 2016; 6. doi:10.1038/srep31606.

10 Schreckenberger M, Siessmeier T, Viertmann A, Landvogt C, Buchholz H-GG, Rolke R et al. The unpleasantness of tonic pain is encoded by the insular cortex. Neurology 2005; 64: 1175-1183.

11 Rainville P, Duncan GH, Price DD, Carrier B, Bushnell MC. Pain affect encoded in human anterior cingulate but not somatosensory cortex. Science 1997; 277: 968-971.

12 Poldrack RA, Fletcher PC, Henson RN, Worsley KJ, Brett M, Nichols TE. Guidelines for reporting an fMRI study. Neuroimage 2008; 40: 409-414.

13 Zeidan F, Martucci KT, a. Kraft R, Gordon NS, McHaffie JG, Coghill RC. Brain mechanisms supporting the modulation of pain by mindfulness meditation. $J$ Neurosci 2011; 31: 5540-5548.

14 Zhou F, Li J, Zhao W, Xu L, Zheng X, Fu M et al. Empathic pain evoked by sensory and emotional-communicative cues share common and process-specific neural representations. Elife 2020; 9. doi:10.7554/eLife.56929.

15 Baliki MN, Chialvo DR, Geha PY, Levy RM, Harden RN, Parrish TB et al. Chronic pain and the emotional brain: specific brain activity associated with spontaneous fluctuations of intensity of chronic back pain. J Neurosci 2006; 26: 12165-12173.

16 Kong J, White NS, Kwong KK, Vangel MG, Rosman IS, Gracely RH et al. Using fMRI to dissociate sensory encoding from cognitive evaluation of heat pain intensity. Hum Brain Mapp 2006; 27: 715-721.

17 Wiech K, Tracey I. Pain, decisions, and actions: a motivational perspective. Front Neurosci 2013; 7: 46.

18 Loggia ML, Juneau M, Bushnell CM. Autonomic responses to heat pain: Heart rate, skin conductance, and their relation to verbal ratings and stimulus intensity. Pain 2011; 152: 592-598.

19 Geuter S, Gamer M, Onat S, Büchel C. Parametric trial-by-trial prediction of pain by easily available physiological measures. Pain 2014; 155: 994-1001.

20 Nickel MM, May ES, Tiemann L, Postorino M, Ta Dinh S, Ploner M. Autonomic responses to tonic pain are more closely related to stimulus intensity than to pain intensity. Pain 2017; 158 : 2129-2136.

21 Mobascher A, Brinkmeyer J, Warbrick T, Musso F, Wittsack HJ, Stoermer R et al. Fluctuations in electrodermal activity reveal variations in single trial brain responses to painful laser stimuli--a 
bioRxiv preprint doi: https://doi.org/10.1101/2021.06.30.450586; this version posted July 1,2021 . The copyright holder for this preprint (which was not certified by peer review) is the author/funder, who has granted bioRxiv a license to display the preprint in perpetuity. It is made available under aCC-BY-NC-ND 4.0 International license.

FMRI/EEG study. Neuroimage 2009; 44: 1081-1092.

22 Rolls ET. The orbitofrontal cortex and emotion in health and disease, including depression. Neuropsychologia 2019; 128: 14-43.

23 Schulz E, Stankewitz A, Winkler AM, Irving S, Witkovský V, Tracey I. Ultra-high-field imaging reveals increased whole brain connectivity underpins cognitive strategies that attenuate pain. Elife 2020; 9. doi:10.7554/eLife.55028.

24 Schulz E, Stankewitz A, Witkovský V, Winkler AM, Tracey I. Strategy-dependent modulation of cortical pain circuits for the attenuation of pain. Cortex. 2019; 113: 255-266.

25 Jenkinson M, Beckmann CF, Behrens TEJ, Woolrich MW, Smith SM. FSL. Neuroimage 2012; 62: 782-790.

26 Griffanti L, Salimi-Khorshidi G, Beckmann CF, Auerbach EJ, Douaud G, Sexton CE et al. ICA-based artefact removal and accelerated FMRI acquisition for improved resting state network imaging. Neuroimage 2014; 95: 232-247.

27 Witkovský V. Estimation, testing, and prediction regions of the fixed and random effects by solving the Henderson's mixed model equations. Measurement science review 2012; 12: 234-248.

28 Michail G, Dresel C, Witkovský V, Stankewitz A, Schulz E. Neuronal Oscillations in Various Frequency Bands Differ between Pain and Touch. Front Hum Neurosci 2016; 10: 182.

29 Wilkinson GN, Rogers CE. Symbolic Description of Factorial Models for Analysis of Variance. $J R$ Stat Soc Ser C Appl Stat 1973; 22: 392-399.

30 Winkler AM, Ridgway GR, Webster MA, Smith SM, Nichols TE. Permutation inference for the general linear model. Neuroimage 2014; 92: 381-397. 